\title{
Chaotic synchronization of memristive neurons: Lyapunov function versus Hamilton function
}

\author{
Marius E. Yamakou(i)
}

Received: 2 March 2020 / Accepted: 20 May 2020 / Published online: 26 June 2020

(C) The Author(s) 2020

\begin{abstract}
In this paper, we consider a 5-dimensional Hindmarsh-Rose neuron model. This improved version of the original model shows rich dynamical behaviors, including a chaotic super-bursting regime. This regime promises a greater information encoding capacity than the standard bursting activity. Based on the Krasovskii-Lyapunov stability theory, the sufficient conditions (on the synaptic strengths and magnetic gain parameters) for stable chaotic synchronization of the model are obtained. Based on Helmholtz's theorem, the Hamilton function of the corresponding error dynamical system is also obtained. It is shown that the time variation of this Hamilton function along trajectories can play the role of the time variation of the Lyapunov function-in determining the stability of the synchronization manifold. Numerical computations indicate that as the synaptic strengths and the magnetic gain parameters change, the time variation of the Hamilton function is always nonzero (i.e., a relatively large positive or negative value) only when the time variation of the Lyapunov function is positive, and zero (or vanishingly small) only when the time variation of the Lyapunov function is also zero. This, therefore, paves an alternative way to determine the stability of synchronization manifolds and can be particularly useful for systems whose Lyapunov function is difficult to con-
\end{abstract}

M. E. Yamakou $(\varangle)$

Angewandte Analysis, Department Mathematik, Friedrich-Alexander-Universität Erlangen-Nürnberg, Cauerstr. 11, 91058 Erlangen, Germany

e-mail: marius.yamakou@fau.de struct, but whose Hamilton function corresponding to the dynamic error system is easier to calculate.

Keywords Neurons - Magnetic flux · Chaotic synchronization - Lyapunov function · Hamilton function

\section{Introduction}

Mathematical modeling, dynamical systems theory, and numerical simulations are important tools for analyzing the complex dynamical behaviors of neural systems. The most biologically plausible mathematical neuron model which describes the generation and transmission of an action potential in neurons was proposed by Hodgkin and Huxley (HH model) in 1952 [1]. Due to the very strong nonlinearity and slow computational speed of the HH model, mathematically simpler and computationally faster neuron models that still capture the qualitative behaviors of the $\mathrm{HH}$ model have been proposed. Some of the popular models includes: FitzHugh-Nagumo (FHN) [2], Hindmarsh-Rose [3], Morris-Lecar [4], and Izhikevich [5] models.

The 2D Hindmarsh-Rose (HR) neuron model [3] is more than ten times faster in computational speed [5] than the $\mathrm{HH}$ model. It is capable of producing some important behaviors such as spiking and sub-threshold oscillations - which are also observed in real biological neurons-upon variations of the model's parameters [5-7]. To capture other dynamical behaviors, such as 
bursting and chaos, observed in real biological neurons, the original 2D HR neuron model has undergone few modifications.

To be able to reproduce bursting activities which mathematically relies on the presence of slow-fast time scales in the model equation, a third equation modeling the dynamics of the slowly varying adaptation ions currents was added to the 2D HR model $[8,9]$. Moreover, this extension of the phase space dimension from 2D to 3D naturally extended the phase space dimension to the minimum required for the occurrence of chaotic dynamics. Because the 3D HR model could mimic, in addition to tonic spiking, bursting and chaotic behaviors observed in real biological neurons, several studies [10-12] have presented a detailed bifurcation analysis of this model, which became very popular in computational neuroscience research in the last decades.

Despite the success of the 3D HR neuron model in reproducing many complex dynamical behaviors of real biological neurons, this model can only capture a relatively small domain of the chaotic regime of these neurons. This failure was corrected by adding an equation for a fourth variable (slower than the slow adaptation current in the 3D model), modeling the dynamics of calcium ions across the intracellular warehouse and the cytoplasm of the neuron [13]. This extension of the phase space dimension from 3D to 4D also increased the complexity of the model; thereby enabling the 4D model to capture the larger chaotic regime of real biological neurons [13-15]. Detailed bifurcation analysis of the 4D HR neuron model can be found in [16-19].

Before the experimental confirmation of the nonnegligible effects of the magnetic flux (generated by the flow of ions across membrane) on the action potential of neurons, all previous studies on the dynamics of neuron models (including the HH, FHN, Izhikevich, 3D, and 4D HR models) have been done without taking into account magnetic flux effects. Recently, M. Lv et al. [20] proposed a modified HR model which takes into account the effect of the magnetic field by adding a variable for the magnetic flux into the 3D HR model. This modified model not only can generate a variety of modes in electric activities by changing the external forcing current and the magnetic flux parameters, but could also be useful in the investigation of the effect of electromagnetic fields on biological tissue [21].

All previous works on the memristive HR neuron model have considered only the $3 \mathrm{D}$ version of the HR model [20,22-27], leading to a 4D model including the memristive (magnetic flux) variable. Thus, for the first time, the memristive property of the $4 \mathrm{D}$ version of the HR model would be considered in this paper. Including the memristive dynamics into the $4 \mathrm{D} \mathrm{HR}$ model leads to a 5D HR model on which the study in this paper will focus on. The aim of this paper is not to present a detailed bifurcation analysis of this improved 5D HR neuron model, but just to show the richer dynamical behavior the model can reproduce. On the other hand, because synchronization of memristive nonlinear systems has become an active area of research and has widespread applications in secure communication and neuromorphic circuits [28,29], the paper mainly focuses on the synchronization dynamics of the memristive 5D HR model.

Using the 5D HR model, our study presents an alternative way to determine the stability of the synchronization manifold of coupled chaotic systems. We show that the time variation of the Hamilton function of the error dynamical system associated to a pair of coupled chaotic 5D HR neurons can be used to determine the stability of the synchronization manifold, just as the time rate of change of Lyapunov function along trajectories of the system would do. It is shown that as the synaptic coupling strengths and memristive gain parameters of the model are varied, we always have a nonzero time variation of this Hamilton function only when the time variation of the Lyapunov function is positive (indicating an unstable synchronized state), and zero (or vanishingly small) only when the time variation of the Lyapunov function is also zero (indicating a stable synchronized state). This indicates that the time variation of the Hamilton function associated with the error dynamical system of coupled oscillators can be used as a stability function.

The rest of this paper is organized as follows: In Sect. 2, we present the improved 5D HR neuron model and show its rich dynamical behavior including spiking, bursting, super-bursting an chaos in time series, phase portraits, and bifurcation diagrams. In Sect. 3, we investigate the chaotic synchronization dynamics of a pair of neurons in the chaotic super-bursting regime and coupled via both time-delayed electrical and chemical synapses. The first part of this section is devoted to the analysis of the stability of the synchronization manifold of the coupled neurons via the Lyapunov stability theory. The second part is devoted to the energy of the synchronization dynamics via the Hamilton function. Sect. 4 deals with numerical simulations, show- 
ing the sign correlation between time variations of the Lyapunov and the Hamilton function of the system. In Sect. 5, we provide summary and concluding remarks.

\section{Mathematical model and dynamics behavior}

The dynamical equation for the memristive 5D HR neuron model is described by

$$
\left\{\begin{aligned}
\frac{\mathrm{d} x}{\mathrm{~d} t}= & -a x^{3}+b x^{2}+y-p z+I_{0} \cos (\Omega t-\psi) \\
& -k_{1} \rho(\phi) x, \\
\frac{\mathrm{d} y}{\mathrm{~d} t}= & c-d x^{2}-y-\sigma w, \\
\frac{\mathrm{d} z}{\mathrm{~d} t}= & r\left[s\left(x+x_{0}\right)-z\right], \\
\frac{\mathrm{d} w}{\mathrm{~d} t}= & \mu\left[\gamma\left(y+y_{0}\right)-\delta w\right], \\
\frac{\mathrm{d} \phi}{\mathrm{d} t}= & x-k_{2} \phi,
\end{aligned}\right.
$$

where $x \in \mathbb{R}$ represents the membrane potential variable, $y \in \mathbb{R}$ is the recovery current variable associated to fast ions, $z \in \mathbb{R}$ is a slow adaption current associated with slow ions, $w \in \mathbb{R}$ represents an even slower process. $I_{0}$ is the amplitude of a harmonic stimulus with frequency $\Omega$ and phase $\psi$. The other constant parameters have standard values: $a=1.0, b=3.0, p=0.99$, $c=1.01, d=5.0128, \sigma=0.0278, r=0.00215$, $s=3.966, x_{0}=1.605, \mu=0.0009, \gamma=3.0$, $y_{0}=1.619, \delta=0.9573$. The parameters $\mu<r \ll 1$ play a very important role in neuron activity; $r$ represents the ratio of timescales between fast and slow fluxes across the neuron's membrane and $\mu$ controls the speed of variation of the slower dynamical process $w$, in particular, the calcium exchange between intracellular warehouse and the cytoplasm $[13,15]$. The $\mu$ timescale induces richer dynamical behaviors including chaos in parameter regimes that the 3D HR shows only periodic dynamics [17].

The fifth variable $\phi \in \mathbb{R}$ describes the magnetic flux across the neuron's cell membrane. The term $\rho(\phi)=\alpha+3 \beta \phi^{2}$ is the memory conductance of a magnetic-flux controlled memristor [30-33], where $\alpha$ and $\beta$ are fixed parameters which we will fix throughout the paper at $\alpha=0.1, \beta=0.02 . k_{1}$ and $k_{2}$ as feedback gain; $k_{1}$ bridges the coupling and modulation on membrane potential $x$ from magnetic field $\phi$, and $k_{2}$ describes the degree of polarization and magnetization by adjusting the saturation of magnetic flux [34]. Following the Faraday's law of electromagnetic induction and the basic properties of a memristor, the term $k_{1} \rho(\phi) v$ could be regarded as additive induction current on the membrane potential. The dependence of electric charge $q$ on magnetic flux $\phi$ is defined by the memory-conductance as follows $[25,35]$

$\rho(\phi)=\frac{d q(\phi)}{d \phi}=\alpha+3 \beta \phi^{2}$.

Moreover, we know that current $i$ is defined by the time rate of charge $q$. Hence, the physical significance for the term $\rho(\phi) v$ could be described as follows

$i=\frac{\mathrm{d} q(\phi)}{\mathrm{d} t}=\frac{\mathrm{d} q(\phi)}{\mathrm{d} \phi} \frac{\mathrm{d} \phi}{\mathrm{d} t}=\rho(\phi) V=k_{1} \rho(\phi) x$,

where the variable $V$ denotes the induced electromotive force, which holds a same physical unit, and parameter $k_{1}$ is the feedback gain. The ion currents of sodium and potassium contribute to the membrane potential and also the magnetic flux across the membrane; thus, a negative feedback term $-k_{2} \phi$ is introduced in the fifth equation of Eq. (1).

We select the amplitude of harmonic forcing current $I_{0}$, its phase $\psi$, and the memristive gain parameters $k_{1}$ and $k_{2}$ as: $I_{0}=1.6, \psi=0.1, k_{1}=1.0$, and $k_{2}=0.5$. In Fig. 1, the time series for membrane potential $x$ under different values of the frequency $\Omega$ of the harmonic forcing are shown. The electrical activity of the 5D HR neuron shows a rich dynamical behavior. In Fig. 1a, the model displays a simple periodic spiking activity with $\Omega=0.2$. In Fig. $1 \mathrm{~b}, \Omega$ decreases to 0.02 and the simple periodic spiking activity changes to the standard bursting activity, with each burst consisting of eleven or twelve spikes. In Fig. 1c, when the frequency is further reduced to $\Omega=0.003$, the standard bursting activity changes to a super-bursting activity, where each super-burst consists of three standard bursts which in turn consist of a different number of spikes. In Fig. 1d, the frequency is increased by a little, i.e., to $\Omega=0.0036$, the pattern of the super-burst changes, with each super-burst consisting this time of only two standard bursts which have different spiking patterns.

The timing precision of the information processing in neural systems is very important because the infor- 

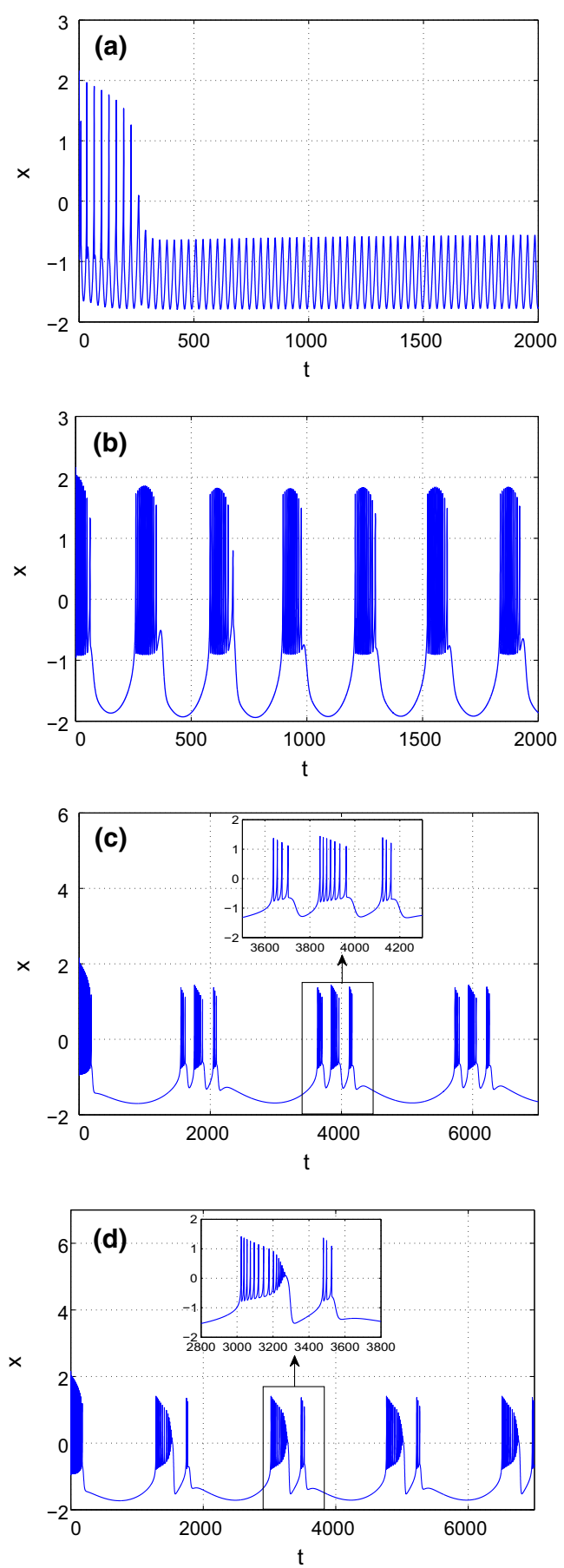

Fig. 1 The time series of the membrane potential $x$. a $\Omega=0.2$, periodic spiking activity. $\mathbf{b} \Omega=0.02$, classical bursting activity. c $\Omega=0.003$, super-bursting activity consisting of three bursts each. d $\Omega=0.0036$, super-bursting activity consisting of only two bursts each. In all panels, the rest parameters are set at: $I_{0}=1.6, k_{1}=1.0, k_{2}=0.5$ mation is encoded at the spiking and bursting times [36]. This means that in our 5D neuron model in a super-bursting regime, one kind of information could be encoded at super-burst time; then a second kind of information encoded at the standard burst times of each of these super-bursts; and then a third kind of information encoded at the spiking time of each standard burst of a super-burst. Whereas in a simple spiking regime, only one kind of information could be encoded at a time. It is well known that bursts provide a more reliable mode of information transfer than spikes [37].

To investigate the dependence of the system's behavior on its parameters-the driving harmonic current $\left(I_{0}, \Omega\right)$ and the memristive gain parameters $\left(k_{1}, k_{2}\right)$ several bifurcation diagrams, each fully traced by its corresponding maximum Lyapunov exponent, have been computed by using the peaks of the spikes in the super-bursting regime of Fig. 1c. The diagrams shown have been chosen to illustrate the general dynamical structure of the memristive 5D HR neuron model. The maximum Lyapunov exponent, $\Lambda_{\max }$, of the model which is defined as

$\Lambda_{\max }=\lim _{\tau \rightarrow \infty} \frac{1}{\tau} \ln [\|L(\tau)\|]$,

where $\|L(\tau)\|=\left(\delta x^{2}+\delta y^{2}+\delta w^{2}+\delta z^{2}+\delta \phi^{2}\right)^{1 / 2}$ is computed numerically by solving, simultaneously, the system in Eq. (1) and its corresponding variational system of equations. Starting from an initial condition, the system of Eq. (1) is numerically integrated with the fourth-order Runge-Kutta algorithm and the data recorded after some transient time. Extensive numerical simulations (not shown) indicate that despite the high dimensionality of the model, it still does not display hyper-chaotic dynamics (i.e., the number of positive Lyapunov exponents is never greater than or equal to two) for the large range of parameter values tested. We either have one positive Lyapunov exponent and the rest negative (chaotic dynamics) or one zero Lyapunov exponent and the rest negative (limit cycle), or all negative Lyapunov exponents (stable fixed point). In this paper, we focus on the chaotic regime shown in Figs. 2 and 3; we therefore only show the most relevant Lyapunov exponent, i.e., the maximum Lyapunov exponent $\Lambda_{\text {max }}$. A strictly negative maximum Lyapunov exponent characterizes an asymptotically stability system, and the more negative the exponent the greater the stability. When $\Lambda_{\max }=0$, we have a marginally stable system with quasi-periodic trajectories. And when 

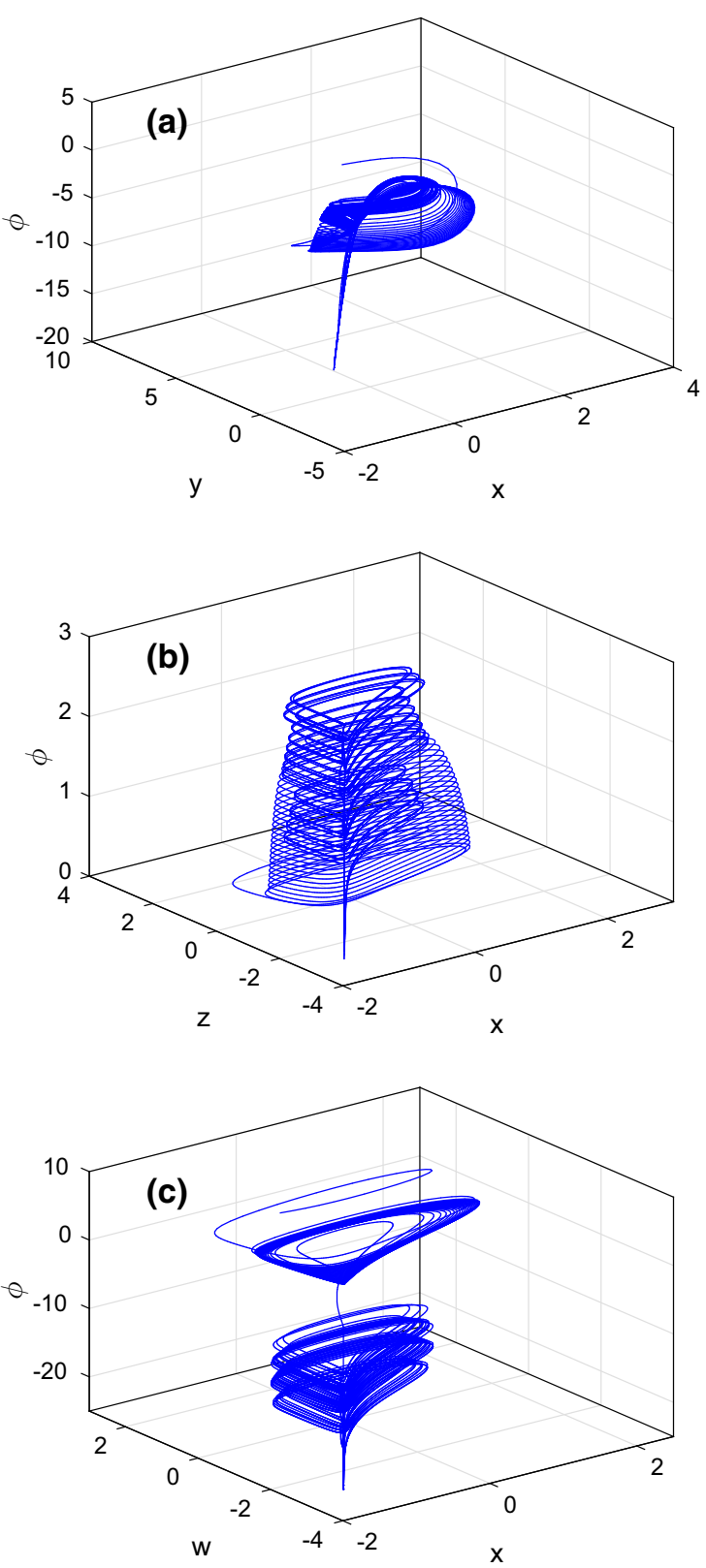

Fig. 2 A bounded chaotic attractor of the improved 5D HR neuron in the super-bursting regime of Fig. 1c, projected onto 3 subspaces of the $5 \mathrm{D}$ phase space. a $x y \phi$-space, $\mathbf{b} x z \phi$-space, $\mathbf{c}$ $x w \phi$-space. The parameters are set at: $I_{0}=1.6, \Omega=0.003$, $k_{1}=1.0, k_{2}=0.5$

$\Lambda_{\max }>0$, the trajectories are unstable, meaning two nearby trajectories would diverge and the evolution of the system becomes sensitive to infinitesimal perturbations of initial conditions and hence chaotic [38].
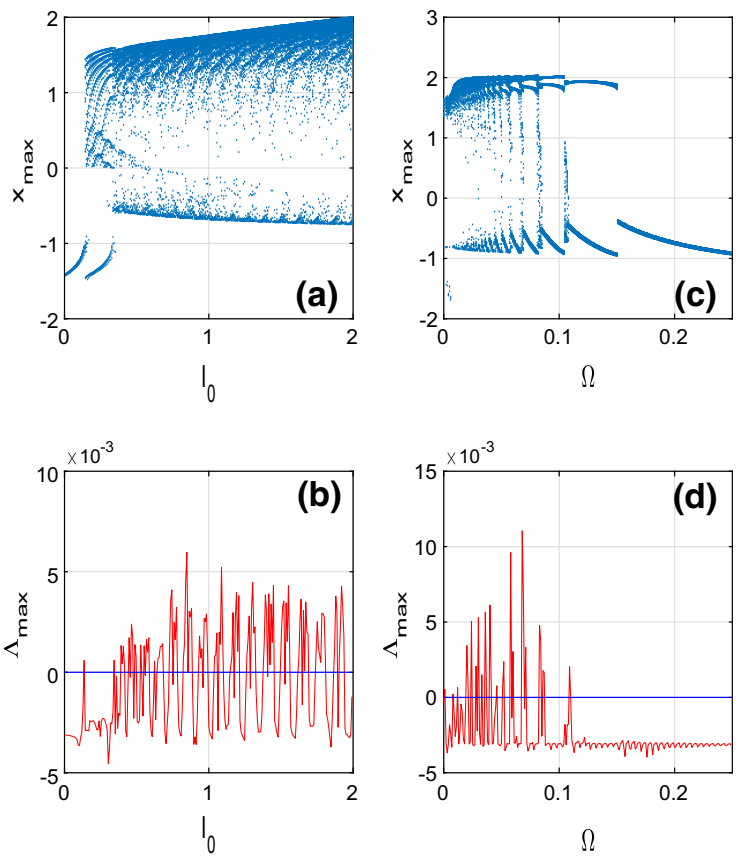

Fig. 3 Bifurcation diagrams each fully traced by its Lyapunov spectrum for the bifurcation parameters $I_{0}$ in (a) and (b) with $\Omega=0.003$; and $\Omega$ in (c) and (d) with $I_{0}=1.6$. In all panels, $k_{1}=1.0$ and $k_{2}=1.2$

Figure $2 \mathrm{a}-\mathrm{c}$ shows the bounded phase space of a chaotic attractor of the 5D memristive HR neuron in a the super-bursting regime, projected onto the $3 \mathrm{D}$ subspaces of the 5D phase space: $x y \phi$-space, $x z \phi$-space, and $x w \phi$-space, respectively.

In Fig. 3a, b, we respectively show a bifurcation diagram and its corresponding Lyapunov spectrum $\Lambda_{\max }$. Different bifurcation sequences occur as amplitude of the external harmonic current is varied. The system dynamics are mainly chaotic, and it is intermingled with thin windows of periodic orbits. In Fig. 3c, d, different bifurcation sequences occur as of the frequency of the external harmonic current is varied. The system dynamics is mainly chaotic for lower frequency values.

In Fig. 4a, b, we respectively show a bifurcation diagram and its corresponding Lyapunov spectrum $\Lambda_{\max }$ for the memristive parameters $k_{1}$. The system dynamics is mainly chaotic for intermediate values of $k_{1}$. In Fig. $4 \mathrm{c}, \mathrm{d}$, chaotic and periodic dynamics are intermingled as $k_{2}$ is varied. In the next section of the paper, the values of the memristive parameters shall be fixed in a chaotic regime. 

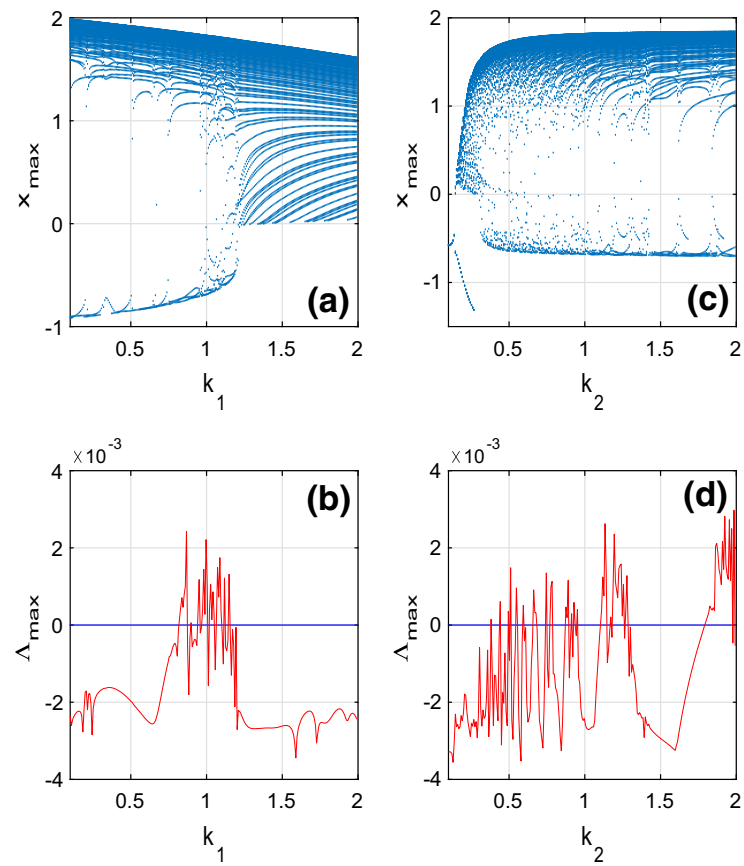

Fig. 4 Bifurcation diagrams each fully traced by its Lyapunov spectrum for the bifurcation parameters $k_{1}$ in $\mathbf{a}$ and $\mathbf{b}$ with fixed at $k_{2}=1.2$; and $k_{2}$ in $\mathbf{c}$ and $\mathbf{d}$ with fixed at $k_{1}=1.0$. In all panels, $I_{0}=1.6$ and $\Omega=0.003$

\section{Synchronization dynamics}

This section deals with the main topic of this paperthe chaotic synchronization dynamics of the coupled 5D HR neuron. Here, we consider a pair of HR neurons coupled via both time-delayed electrical and inhibitory chemical synapses. Electrical and chemical synapses are the two ways through which neurons connect to each other [39]. Electrical synapses connect the cytoplasm of nearby neurons directly, and as a result the transmission of electrical impulses occur relatively quickly. The corresponding functional form of the bidirectional interaction mediated by the electrical synapses is defined as the difference between the membrane potentials of two adjacent neurons. On the other hand, with chemical synapse, the transmission of information takes place via the release of a neurotransmitter. The functional form of this synaptic interaction is considered as a nonlinear sigmoidal input-output function [40]. The system of coupled memristive 5D HR neurons is given as

$$
\left\{\begin{aligned}
\frac{\mathrm{d} x_{i}}{\mathrm{~d} t}= & -a x_{i}^{3}+b x_{i}^{2}+y_{i}-p z_{i}+I_{0} \cos (\Omega t-\psi) \\
& -k_{1}\left(\alpha+3 \beta \phi_{i}^{2}\right) x_{i}+g_{e} \xi_{i}\left(x_{j}-x_{i}\right) \\
& -g_{c}\left(x_{i}-V_{s y n}\right) \sum_{j=1}^{2} \eta_{i j} G\left(x_{i}, x_{j}\right), \\
\frac{\mathrm{d} y_{i}}{\mathrm{~d} t}= & c-d x_{i}^{2}-y_{i}-\sigma w_{i}, \\
\frac{\mathrm{d} z_{i}}{\mathrm{~d} t}= & r\left[s\left(x_{i}+x_{0}\right)-z_{i}\right], \\
\frac{\mathrm{d} w_{i}}{\mathrm{~d} t}= & \mu\left[\gamma\left(y_{i}+y_{0}\right)-\delta w_{i}\right], \\
\frac{\mathrm{d} \phi_{i}}{\mathrm{~d} t}= & x_{i}-k_{2} \phi_{i},
\end{aligned}\right.
$$

with $i, j=\overline{1,2}$. The parameters $g_{e}$ and $g_{c}$ are the electrical and chemical coupling strengths, respectively. The chemical synaptic function is modeled by a sigmoidal nonlinear function $G\left(x_{i}, x_{j}\right)$ defined as: $G\left(x_{i}, x_{j}\right):=G\left(x_{j}\right)=1 /\left[1+\exp \left\{-\lambda\left(x_{j}-\theta_{S}\right)\right\}\right]$, where the parameter $\lambda=10.0$ determines the slope of the function and $\theta_{s}=-0.25$ denotes the synaptic firing threshold. $V_{\text {syn }}$ represents the synaptic reversal potential. For $V_{\text {syn }}<x_{i}$, the chemical synaptic interaction has a depolarizing effect that makes the synapse inhibitory, and for $V_{\text {syn }}>x_{i}$, the synaptic interaction has a hyper-polarizing effect making the synapse excitatory.

For the 5D HR neuron model, the membrane potentials are bounded as $\left|x_{i}(t)\right| \leq 2.0(i=1,2)$ for all times $t$. For the choice of fixed $V_{\text {syn }}=-2.5$ (maintained throughout computations), the term $\left(x_{i}-V_{\text {syn }}\right)$ in Eq. (5) is always positive. So, the inhibitory and excitatory natures of chemical synapses will depend only on the sign in front of the synaptic coupling strengths $g_{c}$. To make the chemical synapse inhibitory, we chose a negative sign. The connectivity matrices $\xi_{i}$ and $\eta_{i j}$ are such that: $\xi_{1}=1$ and $\xi_{2}=-1 ; \eta_{i j}=\eta_{j i}=-1$ if $i \neq j$ and $\eta_{i j}=0$ if $i=j$.

\subsection{Stability of synchronous states and Lyapunov function}

The complete synchronization of the coupled system in Eq. (5) occurs when the two neurons asymptotically exhibit identical behavior, that is 


$$
\left\{\begin{array}{l}
\left\|x_{2}(t)-x_{1}(t)\right\| \rightarrow 0, \\
\left\|y_{2}(t)-y_{1}(t)\right\| \rightarrow 0, \\
\left\|z_{2}(t)-z_{1}(t)\right\| \rightarrow 0, \\
\left\|w_{2}(t)-w_{1}(t)\right\| \rightarrow 0, \\
\left\|\phi_{2}(t)-\phi_{1}(t)\right\| \rightarrow 0,
\end{array}\right.
$$

as $t \rightarrow \infty$, for initial conditions chosen in some neighborhood of the synchronization manifold $\mathcal{M}_{s}$ on which

$$
\left\{\begin{array}{l}
x_{1}(t)=x_{2}(t)=x(t) \\
y_{1}(t)=y_{2}(t)=y(t) \\
z_{1}(t)=z_{2}(t)=z(t) \\
w_{1}(t)=w_{2}(t)=w(t) \\
\phi_{1}(t)=\phi_{2}(t)=\phi(t)
\end{array}\right.
$$

The synchronization solution in Eq. (7) which satisfies the system of dynamical equations

$$
\left\{\begin{aligned}
\frac{\mathrm{d} x}{\mathrm{~d} t}= & -a x^{3}+b x^{2}+y-p z+I_{0} \cos (\Omega t-\psi) \\
& -k_{1}\left(\alpha+3 \beta \phi^{2}\right) x-g_{c}\left(x-V_{s y n}\right) G(x), \\
\frac{\mathrm{d} y}{\mathrm{~d} t}= & c-d x^{2}-y-\sigma w, \\
\frac{\mathrm{d} z}{\mathrm{~d} t}= & r\left[s\left(x+x_{0}\right)-z\right], \\
\frac{\mathrm{d} w}{\mathrm{~d} t}= & \mu\left[\gamma\left(y+y_{0}\right)-\delta w\right], \\
\frac{\mathrm{d} \phi}{\mathrm{d} t}= & x-k_{2} \phi .
\end{aligned}\right.
$$

where the last term, together with its negative sign, in the first equation of Eq. (8) represents a chemical autapse-a negative feedback coupling which models a connection between the soma and the axon of the same neuron [41-44]. $x_{j}-x_{i}=0$ because it is always a solution of the coupled system Eq. (5).

However, this synchronization solution might be stable only under some conditions. To investigate the stability of synchronized states of coupled oscillators, the master stability function approach [45] or the Lyapunov function approach [46] are popularly used. In this work, we choose the latter approach for the simple reason that the Lyapunov function in contrast to the master stability function can also be used to study the stability of other states such as fixed points and limit cycles.

By introducing coordinates transformation defined by directions transverse to the synchronization manifold $\mathcal{M}_{s}$ as

$$
\left\{\begin{array}{l}
e_{x}=x_{2}-x_{1} \\
e_{y}=y_{2}-y_{1} \\
e_{z}=z_{2}-z_{1} \\
e_{w}=w_{2}-w_{1} \\
e_{\phi}=\phi_{2}-\phi_{1}
\end{array}\right.
$$

we obtain the dynamics of the transverse perturbations to the synchronization manifold as

$$
\left\{\begin{aligned}
\frac{\mathrm{d} e_{x}}{\mathrm{~d} t}= & {\left[-3 a x^{2}+2 b x-k_{1} \alpha-3 k_{1} \beta \phi-2 g_{e}\right.} \\
& \left.-g_{c}\left(1+e^{-\lambda\left(x-\theta_{s}\right)}\right)^{-2}\right] e_{x}+e_{y}-p e_{z} \\
& -6 k_{1} \beta x \phi e_{\phi}, \\
\frac{\mathrm{d} e_{y}}{\mathrm{~d} t}= & -2 d x e_{x}-e_{y}-\sigma e_{w}, \\
\frac{\mathrm{d} e_{z}}{\mathrm{~d} t}= & r\left(s e_{x}-e_{z}\right), \\
\frac{\mathrm{d} e_{w}}{\mathrm{~d} t}= & \mu\left(\gamma e_{y}-\delta e_{w}\right), \\
\frac{\mathrm{d} e_{\phi}}{\mathrm{d} t}= & e_{x}-k_{2} e_{\phi} .
\end{aligned}\right.
$$

The following theorem can be obtained.

Theorem 1 The coupled chaotic system in Eq. (5) synchronizes if the five ordered-main sub-determinants in Appendix (A.1) are all strictly positive.

Proof We construct a continuous, positive-definite Lyapunov function $V$ with continuous first partial derivative of the form

$V\left(e_{x}, e_{y}, e_{z}, e_{w}, e_{\phi}\right)=\frac{1}{2}\left[e_{x}^{2}+e_{y}^{2}+e_{z}^{2}+e_{w}^{2}+e_{\phi}^{2}\right]$.

The time derivative of the Lyapunov function $V$ along trajectories of the error dynamical system in Eq. (10) yields

$$
\begin{aligned}
\frac{\mathrm{d} V}{\mathrm{~d} t}= & {\left[-3 a x^{2}+2 b x-k_{1} \alpha-3 k_{1} \beta \phi-2 g_{e}\right.} \\
& \left.-g_{c}\left(1+e^{-\lambda\left(x-\theta_{s}\right)}\right)^{-2}\right] e_{x}^{2}-e_{y}^{2}-r e_{z}^{2}-\mu \delta e_{w}^{2} \\
& -k_{2} e_{\phi}^{2}+(1-2 d x) e_{x} e_{y}+(r s-p) e_{x} e_{z} \\
& +\left(1-6 k_{1} \beta x \phi\right) e_{x} e_{\phi}+(\mu \gamma-\sigma) e_{y} e_{w} .
\end{aligned}
$$

Since chaotic systems have bounded trajectories, there exists a positive constant $J$, such that $|x(t)|<J$ and $|\phi(t)|<J$, thus 


$$
\begin{aligned}
\frac{\mathrm{d} V}{\mathrm{~d} t} \leq & {\left[3|a| J^{2}+2|b| J-\left|k_{1} \alpha\right|+3\left|k_{1} \beta\right| J-2\left|g_{e}\right|\right.} \\
& \left.-\left|g_{c}\right|\left(1+e^{\lambda\left(J+\theta_{s}\right)}\right)^{-2}\right] e_{x}^{2}-e_{y}^{2}-|r| e_{z}^{2}-|\mu \delta| e_{w}^{2} \\
& -\left|k_{2}\right| e_{\phi}^{2}+(1+2|d| J)\left|e_{x}\right|\left|e_{y}\right|+|r s-p|\left|e_{x}\right|\left|e_{z}\right| \\
& +\left(1+6\left|k_{1} \beta\right| J^{2}\right)\left|e_{x}\right|\left|e_{\phi}\right|+|\mu \gamma-\sigma|\left|e_{y}\right|\left|e_{w}\right| .
\end{aligned}
$$

for every points of the attractor and can be compactly written as

$\frac{\mathrm{d} V}{\mathrm{~d} t} \leq-E^{T} \mathbf{M} E$,

where $E^{T}=\left(\left|e_{x}\right|,\left|e_{y}\right|,\left|e_{z}\right|,\left|e_{w}\right|,\left|e_{\phi}\right|\right)$ is a row vector with transpose $E$, and the matrix $\mathbf{M}$ is given by

$\mathbf{M}=\left[\begin{array}{ccccc}A_{x x} & A_{x y} & A_{x z} & 0 & A_{x \phi} \\ A_{y x} & 1 & 0 & -\frac{1}{2}|\mu \gamma-\sigma| & 0 \\ A_{z x} & 0 & |r| & 0 & 0 \\ 0 & -\frac{1}{2}|\mu \gamma-\sigma| & 0 & |\mu \delta| & 0 \\ A_{\phi x} & 0 & 0 & 0 & \left|k_{2}\right|\end{array}\right]$

where

$$
\left\{\begin{aligned}
A_{x y}= & A_{y x}=-\frac{1}{2}(1+2|d| J), \\
A_{x z}= & A_{z x}=-\frac{1}{2}|r s-p|, \\
A_{x \phi}= & A_{\phi x}=-\frac{1}{2}\left(1+6\left|k_{1} \beta\right| J^{2}\right), \\
A_{x x}= & {\left[-3|a| J^{2}-2|b| J+\left|k_{1} \alpha\right|-3\left|k_{1} \beta\right| J\right.} \\
& \left.+2\left|g_{e}\right|+\left|g_{c}\right|\left(1+e^{\lambda\left(J+\theta_{s}\right)}\right)^{-2}\right] .
\end{aligned}\right.
$$

To ensure that the origin of the error dynamical system (i.e., the synchronization manifold) in Eq. (10) is stable, in the Lyapunov sense, $\mathrm{d} V / \mathrm{d} t$ in Eq. (13) should be negative semi-definite. This can only happen if matrix $\mathbf{M}$ in Eq. (14) is positive definite. And $\mathbf{M}$ is positive definite if it satisfies Sylvester's criterion given in "Appendix A.2"-in which case we will have $\mathrm{d} V / \mathrm{d} t \leq 0$. Hence, according to Lyapunov stability theory [46] and Barbalat's lemma [47], all the transverse perturbations decay to the synchronization manifold without any transient growth, i.e., one obtains

$$
\left\{\begin{array}{l}
e_{x}(t) \rightarrow 0 \\
e_{y}(t) \rightarrow 0 \\
e_{z}(t) \rightarrow 0 \\
e_{w}(t) \rightarrow 0 \\
e_{\phi}(t) \rightarrow 0
\end{array}\right.
$$

as $t \rightarrow \infty$. It follows that the coupled chaotic system in Eq. (5) synchronizes when the inequalities in Eq. (A.2) are satisfied. This completes the proof.

\subsection{Synchronization energy and Hamilton function}

Here, we determine the Hamilton energy function $H\left(e_{x}, e_{y}, e_{z}, e_{w}, e_{\phi}\right)$ associated to the error system in Eq. (10). Using this energy function, we analytically evaluate the energy variation of the coupled system. This energy variation is an important measure because it gives us the flow of energy in the process of synchronization, and hence, it can be considered to be the amount of energy per unit time needed to maintain a particular degree of synchrony [48]. We focus on the effects of the synaptic couplings strengths $\left(g_{e}, g_{c}\right)$ and magnetic flux parameters $\left(k_{1}, k_{2}\right)$ on this energy variation and compare it to the time variation of the Lyapunov function previously calculated.

Based on Helmholtz's theorem [49], we express the velocity vector field $F\left(e_{x}, e_{y}, e_{z}, e_{w}, e_{\phi}\right)$ of the error dynamical system in Eq. (10) as the sum of two vector fields: a divergence-free vector and a gradient vector field, i.e., $F=f_{c}+f_{d}$, with the conservative part $f_{c}$, containing the full rotation and the dissipative part, $f_{d}$, containing the whole divergence. A divergence-free vector and a gradient vector field of the error dynamical system in Eq. (10) are given respectively by

$$
\begin{aligned}
f_{c} & =\left(\begin{array}{c}
e_{y}-p e_{z}-6 k_{1} \beta x \phi e_{\phi} \\
-2 d x e_{x}-\sigma e_{w} \\
r s e_{x} \\
\mu \gamma e_{y} \\
e_{x}
\end{array}\right), \\
f_{d} & =\left(\begin{array}{c}
N e_{x} \\
-e_{y} \\
-r e_{z} \\
-\mu \delta e_{w} \\
-k_{2} e_{\phi}
\end{array}\right),
\end{aligned}
$$

where

$$
\begin{aligned}
N & =\left[-3 a x^{2}+2 b x-k_{1} \alpha-3 k_{1} \beta \phi-2 g_{e}\right. \\
& \left.-g_{c}\left(1+e^{-\lambda\left(x-\theta_{s}\right)}\right)^{-2}\right] .
\end{aligned}
$$

For the conservative vector field, the equation

$\nabla H^{T} f_{c}\left(e_{x}, e_{y}, e_{z}, e_{w}, e_{\phi}\right)=0$, 
where $\nabla H$ denotes the transpose gradient of function, $H$ defines a partial differential equation from which a Hamilton energy function (a generalized Hamiltonian) $H\left(e_{x}, e_{y}, e_{z}, e_{w}, e_{\phi}\right)$ can be evaluated (see also [50] for a detailed and general description of the method). Thus, Hamilton energy function of the error dynamical system in Eq. (10) satisfies the partial differential equation given by

$$
\begin{aligned}
& \left(e_{y}-p e_{z}-6 k_{1} \beta x \phi e_{\phi}\right) \frac{\partial H}{\partial e_{x}}-\left(2 d x e_{x}+\sigma e_{w}\right) \frac{\partial H}{\partial e_{y}} \\
& +r s e_{x} \frac{\partial H}{\partial e_{z}}+\mu \gamma e_{y} \frac{\partial H}{\partial e_{w}}+e_{x} \frac{\partial H}{\partial e_{\phi}}=0 .
\end{aligned}
$$

By the method of separation of variables, one solution of Eq. (21) is given by

$$
\begin{aligned}
H= & \left(2 d x+r s p-\mu \gamma \sigma+6 k_{1} \beta x \phi\right) e_{x}^{2}+e_{y}^{2}+p^{2} e_{z}^{2} \\
& +\left(36 k_{1}^{2} \beta^{2} x^{2} \phi^{2}-6 k_{1} \beta \mu \gamma \sigma x \phi+r s p \mu \gamma \sigma\right) e_{\phi}^{2} \\
& +2 \sigma e_{x} e_{w}-2 p e_{y} e_{z}-12 k_{1} \beta x \phi e_{y} e_{\phi}+e_{z} \\
& -r s e_{\phi}+p\left(12 k_{1} \beta x \phi-2 \mu \gamma \sigma\right) e_{z} e_{\phi}+Q,
\end{aligned}
$$

where $Q$ is an arbitrary constant.

Since any positive definite quadratic form can always be a solution for the energy partial differential equation compatible with the generalized Hamiltonian formalism, independently of the system at hand, the same trivial positive definite quadratic form can always be assigned to different chaotic systems. However, assigning the same form of energy function to all chaotic systems fails to reveal the individual features of its own dynamics. Hence, the approach used to obtain the Hamilton energy function $H$ requires additional hypothesis in order to be able to assign to the error dynamical system a specific energy function. This additional hypothesis is set by introducing a connection between the change in the volume of the phase space of the coupled system and time rate of change of energy, as one cannot occur without the other. Hence, since the energy function in Eq. (22) is not unique for the system, we compute the time rate of change of this energy function along trajectories of the error dynamical system. This time rate of change of the Hamilton energy function is related to the divergence of the vector field, $f_{d}$, responsible for the contraction of the volume of the phase space. Thus, the time variation of the Hamilton energy function $H$ associated to the error dynamical system in Eq. (10) now becomes uniquely related to the specific dynamics of the error dynamical system. This energy is dissipated via the dissipative component of the velocity vector field $f_{d}$ according to the equation [50]

$$
\frac{\mathrm{d} H}{\mathrm{~d} t}=\nabla H^{T} f_{d} .
$$

From Eqs. (18), (22) and (23), we obtain the time variation of the Hamilton energy function as

$$
\begin{aligned}
\frac{\mathrm{d} H}{\mathrm{~d} t}= & N\left[\left(4 d x+2 r s p-2 \mu \gamma \sigma+12 k_{1} \beta x \phi\right) e_{x}^{2}+2 \sigma e_{x} e_{w}\right] \\
& -2 e_{y}^{2}+2 p e_{y} e_{z}+12 k_{1} \beta x \phi e_{y} e_{\phi}+2 r p e_{y} e_{z}-2 r p^{2} e_{z}^{2} \\
& -r p\left(12 k_{1} \beta x \phi-2 \mu \gamma \sigma\right) e_{z} e_{\phi}-r e_{z}-2 \mu \delta \sigma e_{x} e_{w} \\
& +12 k_{1} k_{2} \beta x \phi e_{y} e_{\phi}-k_{2} p\left(12 k_{1} \beta x \phi-2 \mu \gamma \sigma\right) e_{z} e_{\phi} \\
& -k_{2}\left(72 k_{1}^{2} \beta^{2} x^{2} \phi^{2}-6 k_{1} \beta \mu \gamma \sigma x \phi+r s p \mu \gamma \sigma\right) e_{\phi}^{2} \\
& +r s k_{2} e_{\phi},
\end{aligned}
$$

where $N$ is given by Eq. (19). Equation (24) gives us an energy function which is unique to the error dynamical system of our coupled neurons, and it gives us information about the energy dissipated during the synchronization dynamics. In the next section, we show (and provide a theoretical explanation) that the time variation of the Hamilton function of the error dynamical associated to the coupled neuron system paves an alternative way of determining the stability of the synchronized state of the system just as the time variation of the Lyapunov function would do.

\section{Numerical simulations and discussion}

In this section, we compare the derivatives of the Lyapunov function and the Hamilton energy function given in Eqs. (12) and (24), respectively, as the synaptic strengths $\left(g_{e}\right.$ and $\left.g_{c}\right)$ and the memristive gain parameters $\left(k_{1}\right.$ and $\left.k_{2}\right)$ vary. To simulate these equations, we simultaneously integrate Eqs. (8) and (10) for a very large time interval using the fourth-order Runge Kutta algorithm. After discarding the transient time, we compute the mean values of $\mathrm{d} V / \mathrm{d} t$ and $\mathrm{d} H / \mathrm{d} t$.

For a weak chemical synaptic strength $g_{c}$, Fig. 5a shows the variations of $\mathrm{d} V / \mathrm{d} t$ and $\mathrm{d} H / \mathrm{d} t$ with respect to electrical synaptic strength $g_{e}$. It is observed that synchronization manifold $\mathcal{M}_{s}$ is always unstable as indicated by $\mathrm{d} V / \mathrm{d} t>0$ (blue curve) for $0 \leq g_{e}<22.5$ and stable as indicated by $\mathrm{d} V / \mathrm{d} t=0.0$ for $22.5 \leq$ $g_{e} \leq 25.0$. For these stable and unstable intervals of $g_{e}$, we have $\mathrm{d} H / \mathrm{d} t>0$ and $\mathrm{d} H / \mathrm{d} t=0.0$ (red curve), respectively. 

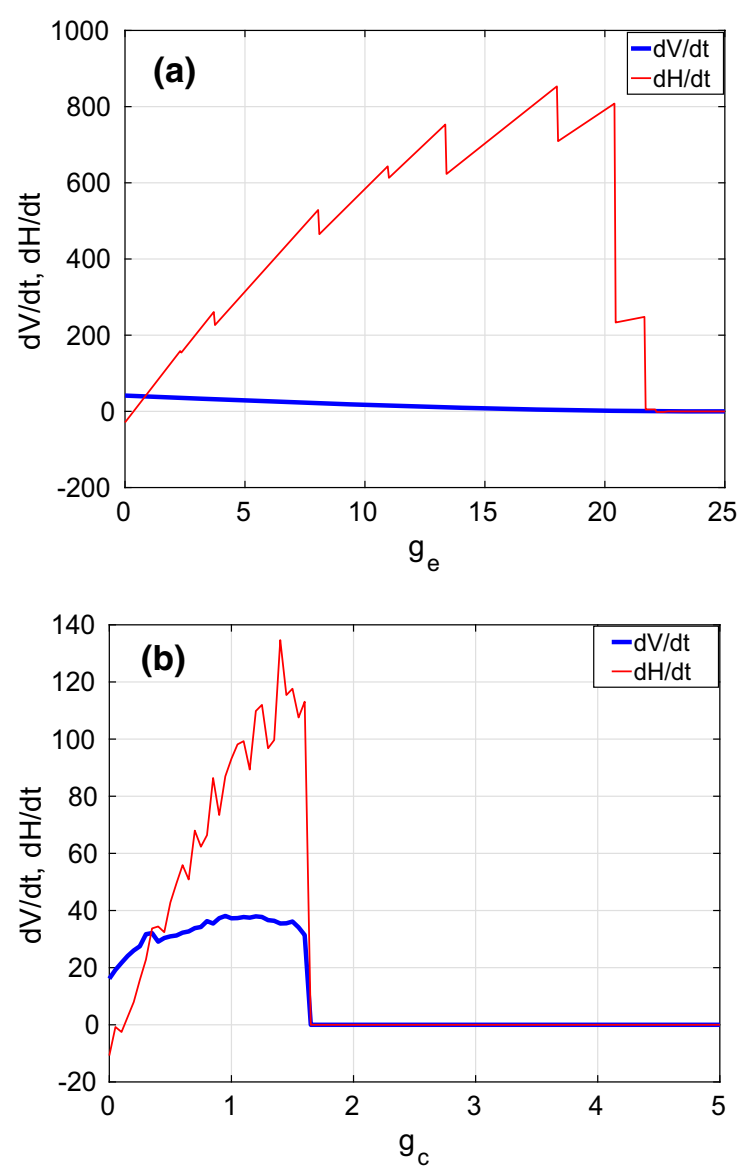

Fig. 5 Variations of $\mathrm{d} V / \mathrm{d} t$ and $\mathrm{d} H / \mathrm{d} t$, respectively, represented by the blue and red curves, with respect to the synaptic strengths $g_{e}$ an $g_{c}$ for fixed memristive gain parameter values. $\mathrm{d} V / \mathrm{d} t>0$, indicating an unstable synchronization manifold $\mathcal{M}_{s}$ only $\mathrm{d} H / \mathrm{d} t<0$. We have $\mathrm{d} V / \mathrm{d} t=0$, for a stable $\mathcal{M}_{s}$, only when $\mathrm{d} H / \mathrm{d} t=0$. In panel (a): $k_{1}=1.0, k_{2}=0.5, g_{c}=1.0$. In panel (b): $k_{1}=1.0, k_{2}=0.5, g_{e}=1.5$

In Fig. 5b, we show the variations of $\mathrm{d} V / \mathrm{d} t$ (blue curve) and $\mathrm{d} H / \mathrm{d} t$ (red curve) with respect to the chemical synaptic strength $g_{c}$ for a weak electrical synaptic strength $g_{e}$. Here, we have that either $\mathrm{d} H / \mathrm{d} t<0.0$ or $\mathrm{d} H / \mathrm{d} t>0.0$ only when $\mathcal{M}_{s}$ is unstable as indicated by $\mathrm{d} V / \mathrm{d} t>0$; and $\mathrm{d} H / \mathrm{d} t=0$ only when $\mathcal{M}_{s}$ is stable as indicated by $\mathrm{d} V / \mathrm{d} t=0$.

In Fig. 6a, b, we show a color-coded global behavior of the $\mathrm{d} V / \mathrm{d} t$ and $\mathrm{d} H / \mathrm{d} t$ with respect to the synaptic coupling strength parameters $g_{e}$ and $g_{c}$ for fixed memristive gain parameters. Here we observe that the sign correlation between $\mathrm{d} V / \mathrm{d} t$ and $\mathrm{d} H / \mathrm{d} t$ persist for all values of the parameters, and not just for particular values. Here, we have $\mathrm{d} H / \mathrm{d} t \neq 0.0$ only when
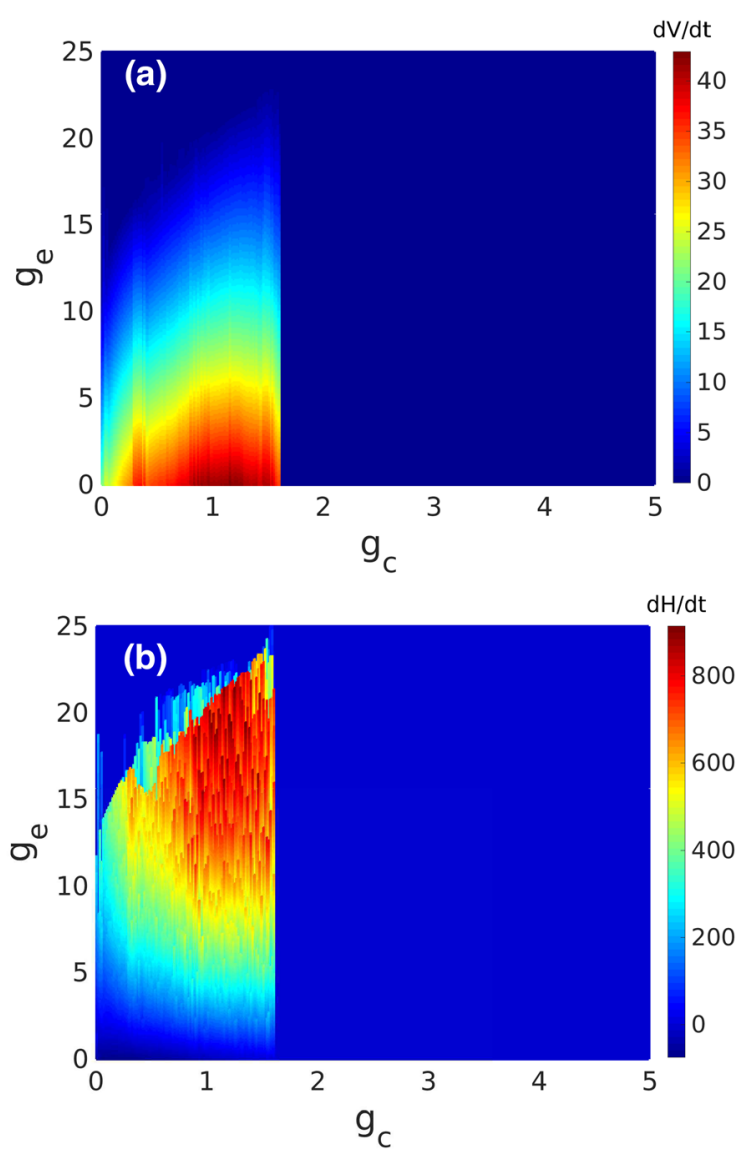

Fig. 6 Color-coded variations of $\mathrm{d} V / \mathrm{d} t$ in (a) and $\mathrm{d} H / \mathrm{d} t$ in (b) with respect to the synaptic coupling strengths for fixed memristive gain parameter values. The synchronization manifold $\mathcal{M}_{s}$ is unstable when $\mathrm{d} V / \mathrm{d} t>0.0$ which occurs only when $\mathrm{d} H / \mathrm{d} t \neq 0.0$; it is stable when $\mathrm{d} V / \mathrm{d} t=0.0$ which occurs only when $\mathrm{d} H / \mathrm{d} t=0.0$ or $\mathrm{d} H / \mathrm{d} t \approx 0.0$ (i.e., vanishingly small). Other parameters: $k_{1}=1.0, k_{2}=0.5$

$\mathrm{d} V / \mathrm{d} t>0.0$, indicating an unstable synchronization manifold. And $\mathrm{d} H / \mathrm{d} t=0.0$ (or $\mathrm{d} H / \mathrm{d} t \approx 0.0$ ) only when $\mathrm{d} V / \mathrm{d} t=0.0$, indicating a stable synchronization manifold.

In Fig. 6a, the color-bar shows the variation of $\mathrm{d} V / \mathrm{d} t$ for the given range of values of $g_{e}$ and $g_{c}$ and indicates that for a sufficiently strong chemical coupling strength, i.e., $g_{c}>1.65$, the synchronization manifold becomes and stays stable, (i.e., $\mathrm{d} V / \mathrm{d} t=0.0$ ) irrespective of the electrical synaptic strength $g_{e}$. And for $g_{c} \leq 1.65$, we have a stable synchronization manifold only when $g_{e}$ is large, i.ie., $g_{e} \geq 22.5$.

In Fig. 6b, where the color-bar shows the variation of $\mathrm{d} H / \mathrm{d} t$, we observed that $\mathrm{d} H / \mathrm{d} t=0$ for $g_{c}>1.65$, irrespective of the value of $g_{e}$ just as with $\mathrm{d} V / \mathrm{d} t$ in 

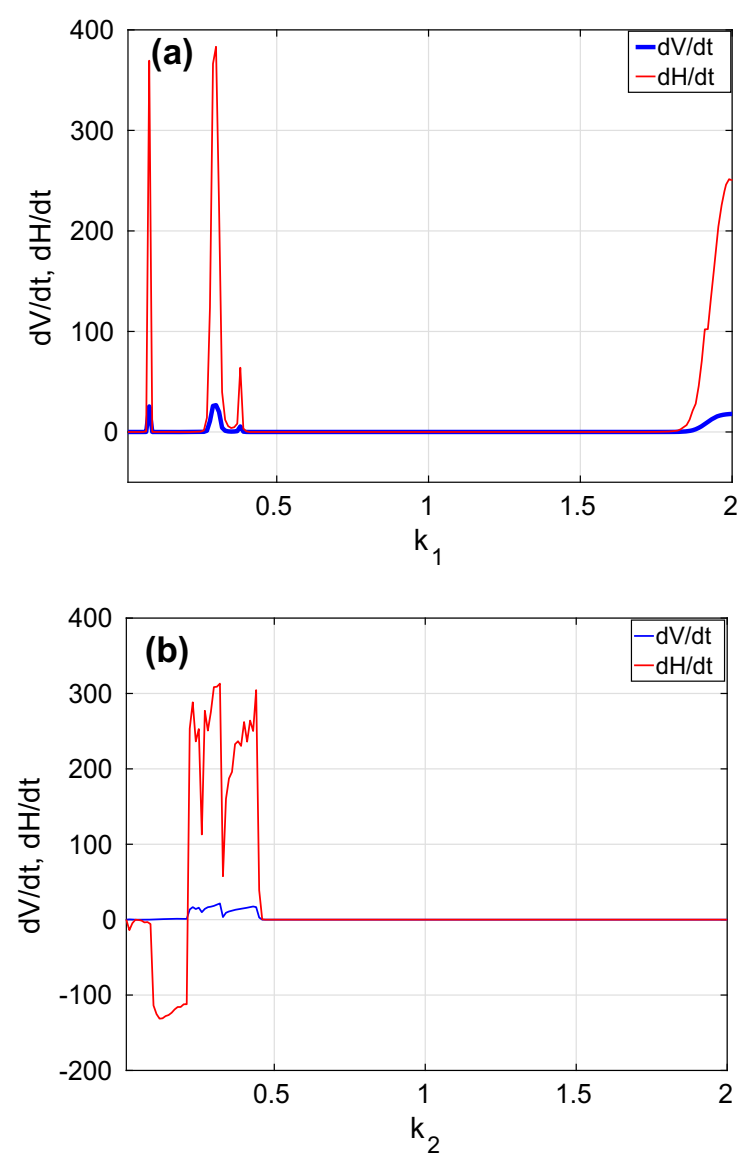

Fig. 7 a and b show the variations of both $\mathrm{d} V / \mathrm{d} t$ and $\mathrm{d} H / \mathrm{d} t$ with $k_{1}$ and $k_{2}$, respectively. In both panels, $\mathrm{d} H / \mathrm{d} t=0$ only when $\mathrm{d} V / \mathrm{d} t=0$, indicating a stable synchronized state, while $\mathrm{d} H / \mathrm{d} t \neq 0$ only when $\mathrm{d} V / \mathrm{d} t>0$, indicating unstable synchronized state. In a $k_{2}=0.5$ and in $\mathbf{b} k_{1}=1.0$. In both panels: $g_{e}=5.0, g_{c}=1.65$

Fig. 6a. For weak chemical coupling, i.e., $g_{c}<1.65$, we have $\mathrm{d} H / \mathrm{d} t \approx 0$ for the same range of values of $g_{e}$ for which $\mathrm{d} V / \mathrm{d} t=0.0 . \mathrm{d} V / \mathrm{d} t>0$ and $\mathrm{d} H / \mathrm{d} t \neq 0.0$ for $0 \leq g_{c} \leq 1.65$ and $0 \leq g_{e}<22.5$, indicating unstable synchronized dynamics.

In Fig. 7, we show the variations of $\mathrm{d} V / \mathrm{d} t$ and $\mathrm{d} H / \mathrm{d} t$ with respect to the memristive gain parameters $k_{1}$ and $k_{2}$. In Fig. 7a, we always have $\mathrm{d} H / \mathrm{d} t>0$ whenever $\mathrm{d} V / \mathrm{d} t>0.0$ for some the values of $k_{1}$, indicating unstable synchronized states; $\mathrm{d} H / \mathrm{d} t=0.0$ whenever $\mathrm{d} V / \mathrm{d} t=0.0$ for the rest of values of $k_{1}$, indicating stable synchronized states. In Fig. 7b, we keep $k_{1}$ fixed and vary $k_{2}$ and we get stable synchronized states with $\mathrm{d} H / \mathrm{d} t=0.0$ and $\mathrm{d} H / \mathrm{d} t \approx 0.0$ whenever $\mathrm{d} V / \mathrm{d} t=0.0$ and unstable synchronized states when $\mathrm{d} V / \mathrm{d} t>0.0$ and $\mathrm{d} H / \mathrm{d} t \neq 0.0$.
To have a global view on the behavior of $\mathrm{d} V / \mathrm{d} t$ and $\mathrm{d} H / \mathrm{d} t$ with respect to the memristive parameters $k_{1}$ and $k_{2}$, we computed these functions in a two-parameter space. Figure 8a, b show a color-coded $\mathrm{d} V / \mathrm{d} t$ and $\mathrm{d} H / \mathrm{d} t$ as a function of $k_{1}$ and $k_{2}$, respectively, at specific values of the coupling strengths $g_{e}=$ 5.0 and $g_{c}=1.65$. In Fig. 8a, we observed synchronized states (the blue regions) where $\mathrm{d} V / \mathrm{d} t=0.0$ corresponds to the yellow and yellowish regions in Fig. $8 \mathrm{~b}$ where $\mathrm{d} H / \mathrm{d} t=0.0$ and $\mathrm{d} H / \mathrm{d} t \approx 0.0$. And wherever $\mathrm{d} V / \mathrm{d} t>0$ in Fig. 8a, we have $\mathrm{d} H / \mathrm{d} t \neq 0$ (i.e., either positive or negative), indicating unsynchronized states.

In Fig. 8c, d, we double the strength of each synaptic coupling. Here, panels (c) and (d) also show a colorcoded $\mathrm{d} V / \mathrm{d} t$ and $\mathrm{d} H / \mathrm{d} t$ as a function of $k_{1}$ and $k_{2}$, respectively. In panel (c), we only have $\mathrm{d} V / \mathrm{d} t=0.0$ (stable synchronized state) in blue regions, whenever $\mathrm{d} H / \mathrm{d} t=0.0$ or $\mathrm{d} H / \mathrm{d} t \approx 0.0$ in panel $(\mathbf{d})$ in green regions.

A theoretical explanation for this sign correlation between time rate of change of the Lyapunov $\mathrm{d} V / \mathrm{d} t$ and Hamilton $\mathrm{d} H / \mathrm{d} t$ functions, and hence, the ability of $\mathrm{d} H / \mathrm{d} t$ to also indicate whether or not a synchronized state is stable is the following: First, we notice that $\mathrm{d} H / \mathrm{d} t$ can be either positive or negative (only when $\mathrm{d} V / \mathrm{d} t>0$, indicating unstable synchronized states).

When $\mathrm{d} V / \mathrm{d} t>0$, the chaotic system (i.e., the coupled neurons) initially located outside its synchronization manifold would gain $(\mathrm{d} H / \mathrm{d} t>0)$ or lose energy $(\mathrm{d} H / \mathrm{d} t<0)$ in its movement towards synchronization manifold, where $\mathrm{d} V / \mathrm{d} t=0$ (indicating a stable synchronous state) and $\mathrm{d} H / \mathrm{d} t=0$ or $\mathrm{d} H / \mathrm{d} t \approx 0$ (i.e., or vanishingly small). This is so because, on the synchronization manifold, the trajectory will repeatedly return to arbitrarily close states in the bounded phase space of the chaotic attractor and as a result to arbitrarily close energy values. Hence, on synchronization manifold the average time rate of Hamilton energy function will be zero, i.e., $\mathrm{d} H / \mathrm{d} t=0$ or vanishingly small, i.e., $\mathrm{d} H / \mathrm{d} t \approx 0$. This implies that, in general, all the different regimes of synchronization that the two neurons attain at different values of the system's parameter would occur at zero (or very low) net dissipation of energy (i.e., at $\mathrm{d} H / \mathrm{d} t=0$ or $\mathrm{d} H / \mathrm{d} t \approx 0$ ).

But, there could be ranges of parameter values where the activity of the coupled system is more demanding energetically, that is when $\mathrm{d} H / \mathrm{d} t \neq 0$ which occurs only when $\mathrm{d} V / \mathrm{d} t>0$, indicating unstable synchronized states. This means that when the neurons are 

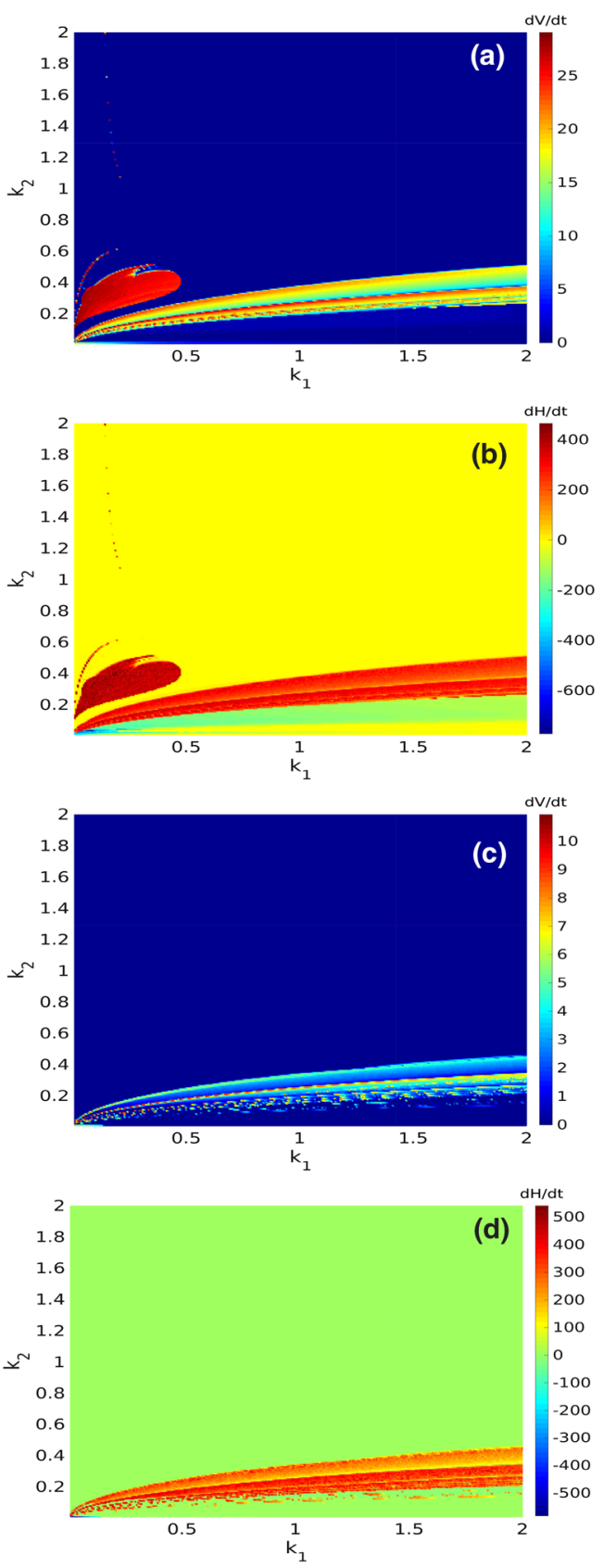

Fig. 8 Color-coded variations of $\mathrm{d} V / \mathrm{d} t$ in (a) and the corresponding $\mathrm{d} H / \mathrm{d} t$ in (b) with respect to $k_{1}$ and $k_{2}$ at $g_{e}=5.0, g_{c}=$ 1.65. $\mathbf{c}$ and $\mathbf{d}$ respectively show the color-coded variations of $\mathrm{d} V / \mathrm{d} t$ and $\mathrm{d} H / \mathrm{d} t$ with respect to $k_{1}$ and $k_{2}$ at couplings strengths two times stronger than in (a) and (b): $g_{e}=10.0, g_{c}=3.3$. In both cases, unstable synchronized states emerge at values of $k_{1}$ and $k_{2}$ for which $\mathrm{d} V / \mathrm{d} t>0$ and $0<\mathrm{d} H / \mathrm{d} t<0$, while the stable synchronized states occur at $\mathrm{d} V / \mathrm{d} t \leq 0$ which correspond to $\mathrm{d} H / \mathrm{d} t=0$ or $\mathrm{d} H / \mathrm{d} t \approx 0$ out of the synchronization manifold, a nonzero energy dissipation $(\mathrm{d} H / \mathrm{d} t \neq 0)$ is thus necessary to drive the coupled neurons to the synchronization manifold where the net energy dissipated become zero or very low $(\mathrm{d} H / \mathrm{d} t=0$ or $\mathrm{d} H / \mathrm{d} t \approx 0)$ and where the time rate of change of the Lyapunov function is also zero $(\mathrm{d} V / \mathrm{d} t=0)$-indicating a stable synchronized state following Krasovskii-Lyapunov stability theory. Hence, the time rate of change of the Hamilton function of an error dynamical system associated with a coupled system can be used as a synchronization stability function.

\section{Summary and concluding remarks}

In this paper, the synchronization dynamics of two coupled 5D Hindmarsh-Rose neurons is investigated. We considered a pair of neurons coupled via both an instantaneous electrical and inhibitory chemical synapses. Using the Krasovskii-Lyapunov stability theory, we prove that the synchronization manifold of the coupled neurons can be stable for suitable values of the electrical and chemical coupling strengths $g_{e}$ and $g_{c}$, and the memristive gain parameters $k_{1}$ and $k_{2}$. Moreover, we used Helmholtz's theorem to calculate the Hamilton energy function associated with the error dynamical of the system of the coupled neurons. Numerical computations indicated that we always have a nonzero $(\mathrm{d} H / \mathrm{d} t \neq 0)$ time variation of the Hamilton function only when the time variation of the Lyapunov function is positive $(\mathrm{d} V / \mathrm{d} t>0)$, and zero $(\mathrm{d} H / \mathrm{d} t=0)$ (or vanishingly small, $\mathrm{d} H / \mathrm{d} t \approx 0$ ) only when the time variation of the Lyapunov function is also zero $(\mathrm{d} V / \mathrm{d} t=0)$. Thus, the time variation of the Hamilton energy function of the error dynamical system associated with a coupled system can be used as a stability function for synchronization. A theoretical explanation of this sign relationships between $\mathrm{d} H / \mathrm{d} t$ and $\mathrm{d} V / \mathrm{d} t$ is also given.

This result which might be also useful for general engineering purposes paves an alternative way of determining the stability of the synchronized states in coupled systems from an energy perspective without necessarily having to construct a Lyapunov function which might be a difficult task for systems modeled with more complicated mathematical equations.

The work presented in this paper could be extended in two directions. First, by considering synaptic and/or channel noise (e.g., Gaussian white noise or non- 
Gaussian colored noises), due to their presence and relevance in real neural dynamics. And secondly, by investigating the variation of the synchronization energy between the layers of a multiplex neural network-a relevant network structure ubiquitous in the brain.

Acknowledgements Open Access funding provided by Projekt DEAL. The author gratefully acknowledges the financial support from the Lehrstuhl für Angewandte Analysis (Alexander von Humboldt-Professur), Department Mathematik, FriedrichAlexander-Universität Erlangen-Nürnberg, Germany.

\section{Compliance with ethical standards}

Conflict of interest The author declares that there is no conflict of interest in relation to this article.

Open Access This article is licensed under a Creative Commons Attribution 4.0 International License, which permits use, sharing, adaptation, distribution and reproduction in any medium or format, as long as you give appropriate credit to the original author(s) and the source, provide a link to the Creative Commons licence, and indicate if changes were made. The images or other third party material in this article are included in the article's Creative Commons licence, unless indicated otherwise in a credit line to the material. If material is not included in the article's Creative Commons licence and your intended use is not permitted by statutory regulation or exceeds the permitted use, you will need to obtain permission directly from the copyright holder. To view a copy of this licence, visit http://creativecommons.org/licenses/ by/4.0/.

\section{Appendix}

The five ordered-main sub-determinants of matrix $\mathbf{M}$ in Eq. (14) are given by

$$
\left\{\begin{aligned}
\mathbf{D}_{\mathbf{1}}= & \mathbf{A}_{\mathbf{x x}}=\left[-3|a| J^{2}-2|b| J+\left|k_{1} \alpha\right|\right. \\
& \left.-3\left|k_{1} \beta\right| J+2\left|g_{e}\right|+\left|g_{c}\right|\left(1+e^{\lambda\left(J+\theta_{s}\right)}\right)^{-2}\right] \\
\mathbf{D}_{\mathbf{2}}= & \mathbf{D}_{\mathbf{1}}-\frac{1}{4}-|d| J-|d|^{2} J^{2} \\
\mathbf{D}_{\mathbf{3}}= & |r|\left(\mathbf{D}_{\mathbf{2}}-\frac{1}{4|r|}|r s-p|^{2}\right), \\
\mathbf{D}_{\mathbf{4}}= & |\mu \delta| \mathbf{D}_{\mathbf{3}}-\frac{1}{4}|r||\mu \gamma-\sigma|^{2} \mathbf{D}_{\mathbf{1}} \\
& +\frac{1}{16}|r s-p|^{2}|\mu \gamma-\sigma|^{2}, \\
\mathbf{D}_{\mathbf{5}}= & \left|k_{2}\right| \mathbf{D}_{\mathbf{4}}+\frac{|r|}{2}\left(9\left|\beta k_{1}\right|^{2} J^{4}+3\left|\beta k_{1}\right| J^{2}+\frac{1}{8}\right) \\
\times & \left(|\mu \gamma-\sigma|^{2}-4|\mu \delta|\right) .
\end{aligned}\right.
$$

It is easy to check that, for the given values of $a, b, d, r$, $s, p, \mu, \alpha, \delta, \gamma, \sigma$, and for suitable values of $g_{e}, g_{c}, k_{1}$, and $k_{2}$, we can always have

$\mathbf{D}_{\mathbf{i}}>0, \mathbf{i}=\{1,2,3,4,5\}$,

which makes the matrix $\mathbf{M}$ positive definite.

\section{References}

1. Hodgkin, A.L., Huxley, A.F.: A quantitative description of membrane current and its application to conduction and excitation in nerve. J. Physiol. 117(4), 500-544 (1952)

2. FitzHugh, R.: Impulses and physiological states in theoretical models of nerve membrane. Biophys. J. 1(6), 445-466 (1961)

3. Hindmarsh, J., Rose, R.: A model of the nerve impulse using two first-order differential equations. Nature 296(5853), 162-164 (1982)

4. Morris, C., Lecar, H.: Voltage oscillations in the barnacle giant muscle fiber. Biophys. J. 35(1), 193-213 (1981)

5. Izhikevich, E.M.: Which model to use for cortical spiking neurons? IEEE Trans. Neural Netw. 15(5), 1063-1070 (2004)

6. Wang, Z., Shi, X.: Lag synchronization of multiple iden- tical Hindmarsh-Rose neuron models coupled in a ring structure. Nonlinear Dyn. 60(3), 375-383 (2010)

7. Djati, N.S.G.: Bidirectional chaotic synchronization of Hindmarsh-Rose neuron model. Appl. Math. Sci. 5(54), 2685-2695 (2011)

8. Hindmarsh, J.L., Rose, R.: A model of neuronal bursting using three coupled first order differential equations. Proc. R. Soc. Lond. Ser. B. Biol. Sci. 221(1222), 87-102 (1984)

9. Coombes, S., Bressloff, P.C.: Bursting: The Genesis of Rhythm in the Nervous System. World Scientific, Singapore (2005)

10. Storace, M., Linaro, D., de Lange, E.: The Hindmarsh-Rose neuron model: bifurcation analysis and piecewise linear approximations. Chaos Interdiscip. J. Nonlinear Sci. 18(3), 033128 (2008)

11. González-Miranda, J.M.: Observation of a continuous interior crisis in the Hindmarsh-Rose neuron model. Chaos Interdiscip. J. Nonlinear Sci. 13(3), 845-852 (2003)

12. González-Miranda, J.: Complex bifurcation structures in the Hindmarsh-Rose neuron model. Int. J. Bifurcat. Chaos 17(09), 3071-3083 (2007)

13. Selverston, A.I., Rabinovich, M.I., Abarbanel, H.D., Elson, R., Szüics, A., Pinto, R.D., Huerta, R., Varona, P.: Reliable circuits from irregular neurons: a dynamical approach to understanding central pattern generators. J. Physiol. Paris 94(5-6), 357-374 (2000)

14. De Lange, E., Hasler, M.: Predicting single spikes and spike patterns with the Hindmarsh-Rose model. Biol. Cybern. 99(4-5), 349 (2008)

15. Pinto, R.D., Varona, P., Volkovskii, A., Szücs, A., Abarbanel, H.D., Rabinovich, M.I.: Synchronous behavior of two coupled electronic neurons. Phys. Rev. E 62(2), 2644 (2000)

16. Megam Ngouonkadi, E., Fotsin, H.B., Louodop Fotso, P.: The combined effect of dynamic chemical and electrical 
synapses in time-delay-induced phase-transition to synchrony in coupled bursting neurons. Int. J. Bifurcat. Chaos 24(05), 1450069 (2014)

17. Ngouonkadi, E.M., Fotsin, H., Fotso, P.L., Tamba, V.K., Cerdeira, H.A.: Bifurcations and multistability in the extended Hindmarsh-Rose neuronal oscillator. Chaos Solitons Fractals 85, 151-163 (2016)

18. Falcke, M., Huerta, R., Rabinovich, M.I., Abarbanel, H.D., Elson, R.C., Selverston, A.I.: Modeling observed chaotic oscillations in bursting neurons: the role of calcium dynamics and IP 3. Biol. Cybern. 82(6), 517-527 (2000)

19. Rabinovich, M.I., Pinto, R., Abarbanel, H.D., Tumer, E., Stiesberg, G., Huerta, R., Selverston, A.I.: Recovery of hidden information through synaptic dynamics. Netw. Comput. Neural Syst. 13(4), 487-501 (2002)

20. Lv, M., Wang, C., Ren, G., Ma, J., Song, X.: Model of electrical activity in a neuron under magnetic flow effect. Nonlinear Dyn. 85(3), 1479-1490 (2016)

21. Wu, F., Wang, C., Xu, Y., Ma, J.: Model of electrical activity in cardiac tissue under electromagnetic induction. Sci. Rep. 6, 28 (2016)

22. Bao, B.C., Hu, A.H., Bao, H., Xu, Q., Chen, M., Wu, H.G.: Three-dimensional memristive Hindmarsh-Rose neuron model with hidden coexisting asymmetric behaviors. Complexity 2018, 3872573 (2018)

23. Liu, Y., Nazarimehr, F., Khalaf, A.J.M., Alsaedi, A., Hayat, T.: Detecting bifurcation points in a memristive neuron model. Eur. Phys. J. Spec. Top. 228(10), 1943-1950 (2019)

24. Panahi, S., Jafari, S., Khalaf, A.J.M., Rajagopal, K., Pham, V.T., Alsaadi, F.E.: Complete dynamical analysis of a neuron under magnetic flow effect. Chin. J. Phys. 56(5), 2254-2264 (2018)

25. Lv, M., Ma, J.: Multiple modes of electrical activities in a new neuron model under electromagnetic radiation. Neurocomputing 205, 375-381 (2016)

26. Xu, Y., Jia, Y., Ma, J., Hayat, T., Alsaedi, A.: Collective responses in electrical activities of neurons under field coupling. Sci. Rep. 8(1), 1349 (2018)

27. Usha, K., Subha, P.: Hindmarsh-Rose neuron model with memristors. Biosystems 178, 1-9 (2019)

28. Abdurahman, A., Jiang, H., Teng, Z.: Finite-time synchronization for memristor-based neural networks with timevarying delays. Neural Netw. 69, 20-28 (2015)

29. Abdurahman, A., Jiang, H., Rahman, K.: Function projective synchronization of memristor-based cohen-grossberg neural networks with time-varying delays. Cognit. Neurodyn. 9(6), 603-613 (2015)

30. Muthuswamy, B.: Implementing memristor based chaotic circuits. Int. J. Bifurcat. Chaos 20(05), 1335-1350 (2010)

31. Bao, B., Liu, Z., Xu, J.: Steady periodic memristor os- cillator with transient chaotic behaviours. Electron. Lett. 46(3), 237-238 (2010)

32. Li, Q., Zeng, H., Li, J.: Hyperchaos in a 4d memristive circuit with infinitely many stable equilibria. Nonlinear Dyn. 79(4), 2295-2308 (2015)

33. Volos, C.K., Kyprianidis, I.M., Stouboulos, I.N., TleloCuautle, E., Vaidyanathan, S.: Memristor: a new concept in synchronization of coupled neuromorphic circuits. J. Eng. Sci. Technol. Rev. 8(2), 157-173 (2015)
34. Ma, J., Wang, Y., Wang, C., Xu, Y., Ren, G.: Mode selection in electrical activities of myocardial cell exposed to electromagnetic radiation. Chaos Solitons Fractals 99, 219-225 (2017)

35. Hong, Q.H., Zeng, Y.C., Li, Z.J.: Design and simulation of chaotic circuit for flux-controlled memristor and chargecontrolled memristor. Acta Phys. Sin. 62(23), 230502 (2013)

36. Pei, X., Wilkens, L., Moss, F.: Noise-mediated spike timing precision from aperiodic stimuli in an array of HodgkinHuxley-type neurons. Phys. Rev. Lett. 77(22), 4679 (1996)

37. Lisman, J.E.: Bursts as a unit of neural information: making unreliable synapses reliable. Trends Neurosci. 20(1), 38-43 (1997)

38. Kwuimy, C.K., Woafo, P.: Dynamics, chaos and synchronization of self-sustained electromechanical systems with clamped-free flexible arm. Nonlinear Dyn. 53(3), 201-213 (2008)

39. Pereda, A.E.: Electrical synapses and their functional interactions with chemical synapses. Nat. Rev. Neurosci. 15(4), 250-263 (2014)

40. Greengard, P.: The neurobiology of slow synaptic transmission. Science 294(5544), 1024-1030 (2001)

41. Wang, C., Guo, S., Xu, Y., Ma, J., Tang, J., Alzahrani, F., Hobiny, A.: Formation of autapse connected to neuron and its biological function. Complexity 2017, 5436737 (2017)

42. Qu, L., Du, L., Zhang, H., Cao, Z., Deng, Z.: Regulation of chemical autapse on an FHN-ML neuronal system. Int. J. Bifurcat. Chaos 29(14), 1950202 (2019)

43. Jia, B.: Negative feedback mediated by fast inhibitory autapse enhances neuronal oscillations near a hopf bifurcation point. Int. J. Bifurcat. Chaos 28(02), 1850030 (2018)

44. Yilmaz, E., Ozer, M., Baysal, V., Perc, M.: Autapse-induced multiple coherence resonance in single neurons and neuronal networks. Sci. Rep. 6, 30914 (2016)

45. Pecora, L.M., Carroll, T.L.: Master stability functions for synchronized coupled systems. Phys. Rev. Lett. 80(10), 2109 (1998)

46. Krasovskii, N.N.: Stability of Motion, vol. 2. Stanford University Press, Stanford (1963)

47. Popov, V.M., Georgescu, R.: Hyperstability of Control Systems. Springer, Berlin (1973)

48. Torrealdea, F., d'Anjou, A., Graña, M., Sarasola, C.: Energy aspects of the synchronization of model neurons. Phys. Rev. E 74(1), 011905 (2006)

49. Kobe, D.H.: Helmholtzs theorem revisited. Am. J. Phys. 54(6), 552-554 (1986)

50. Wang, C.-N., Wang, Y., Ma, J.: Calculation of hamilton energy function of dynamical system by using helmholtz theorem. Acta Phys. Sin. 65, 24 (2016)

Publisher's Note Springer Nature remains neutral with regard to jurisdictional claims in published maps and institutional affiliations. 